\title{
ОБ ОДНОМ ИНТЕГРАЛЬНОМ УРАВНЕНИИ В ТЕОРИИ ОПЕРАТОРОВ ПРЕОБРАЗОВАНИЯ
}

\author{
(C) 2020 г. С. М. Ситник \\ 308002 Белгород, пр-т Победы, 40, НИУ БелГУ, Россия \\ e-mail: sitnik@bsu.edu.ru \\ Поступила в редакцию 15.02.2020 г. \\ Переработанный вариант 15.02.2020 г. \\ Принята к публикации 09.04.2020 г.
}

\begin{abstract}
Рассматриваются представления в интегральном виде решений одного дифференциального уравнения с особенностями в коэффициентах, содержащего оператор Бесселя, возмущенный некоторым потенциалом. Доказывается существование интегральных представлений определенного вида для указанных решений методом последовательных приближений с использованием операторов преобразования. При этом допускаются потенциалы с сильными сингулярностями в начале координат. По сравнению с известными результатами функция Римана выражается не через общую гипергеометрическую функцию, а более конкретно через функцию Лежандра, что позволяет избежать неизвестных постоянных в оценках. Библ. 30.
\end{abstract}

Ключевые слова: оператор преобразования, функция Римана, гипергеометрическая функция Гаусса, функция Лежандра, сингулярный потенциал.

DOI: $10.31857 / \mathrm{S} 0044466920080141$

\section{1. ВВЕДЕНИЕ И ПОСТАНОВКА ЗАДАЧИ}

Рассмотрим задачу о построении интегрального представления определенного вида для решений дифференциального уравнения

$$
B_{\alpha} u(x)-q(x) u(x)=0, \quad x>0,
$$

где $B_{\alpha}-$ оператор Бесселя вида

$$
B_{\alpha} u=u^{\prime \prime}(x)+\frac{2 \alpha}{x} u^{\prime}(x), \quad \alpha>0, \quad x>0 .
$$

Данная задача решается методом операторов преобразования. Для этого достаточно построить оператор преобразования $\mathbf{P}_{\alpha}$ типа Пуассона вида

$$
\mathbf{P}_{\alpha} u(x)=u(x)+\int_{x}^{\infty} P(x, t) u(t) d t
$$

с некоторым ядром $P(x, t)$, который сплетает операторы $B_{\alpha}$ и $B_{\alpha}-q(x)$ по формуле

$$
B_{\alpha} \mathbf{P}_{\alpha} u=\mathbf{P}_{\alpha}\left(B_{\alpha}-q(x)\right) u
$$

на функциях $u \in C^{2}(0, \infty)$. В результате получится формула, выражающая решения уравнения (1) со спектральным параметром вида

$$
B_{\alpha} u(x)-q(x) u(x)=\lambda^{2} u(x), \quad x>0
$$

через решения невозмущенного уравнения, т.е. через функции Бесселя. При этом спектральный параметр $\lambda$ не влияет на вид линейных операторов преобразования, ядра которых от него не зависят. Этот подход отражает одно из основных применений операторов преобразования - выражение решений более сложных дифференциальных уравнений через подобные более простые.

Теория операторов преобразования - это важный раздел современной математики, имеющий многочисленные приложения, см. [1]-[6]. Возможность представления вида (3) с достаточно “хорошим” ядром $P$ для широкого класса потенциалов $q(x)$ лежит в основе классических ме- 
тодов решения обратных задач квантовой теории рассеяния [7], [8]. Для уравнений Штурма-Лиувилля операторы преобразования вида (3) были впервые построены Б.Я. Левиным, см. [1]-[6].

Операторы преобразования для оператора Бесселя типа Сонина и Пуассона были введены Дельсартом, впервые на русском языке их теория была изложена и развита в известной работе Б.М. Левитана [9]. Затем в ряде работ были рассмотрены и операторы преобразования со свойством (4) для переменных потенциалов. При этом одновременно рассматриваются и обратные к (3) операторы преобразования типа Сонина $S_{\alpha}$, которые удовлетворяют сплетающему соотношению

$$
S_{\alpha}\left(B_{\alpha}-q(x)\right) u=B_{\alpha} S_{\alpha} u
$$

на подходящих функциях.

Оригинальная методика для построения операторов преобразования для возмущенного уравнения Бесселя на полуоси была разработана В.В. Сташевской [10], что позволило ей включить в рассмотрение сингулярные потенциалы с оценкой в нуле $|q(x)| \leq c x^{-3 / 2+\varepsilon}, \varepsilon>0$, при целых $\alpha$, эта методика в дальнейшем получила широкое развитие. Случай непрерывной $q, \alpha>0$ подробно рассмотрен в работах А.С. Сохина [11], [12], а также ряда других авторов (см. подробнее [5], [6]). Операторы преобразования с “плохими” потенциалами-распределениями из определенного класса рассматривались в [8]. В.В. Кравченко был развит специальный метод для представления ядер операторов преобразования в виде рядов (метод SPPS - Spectral Parameter Power Series) [13], [14], этот метод оказался хорошо приспособленным для численного решения прикладных задач и компьютерного моделирования, в том числе для решения прямых и обратных спектральных задач [15], [16]. Результаты автора для некоторых частных случаев рассматриваемой задачи о построении операторов преобразования (3), (4) ранее опубликованы в [17], [18].

Во многих математических и физических задачах необходимо рассматривать сильно сингулярные потенциалы, например, допускающие произвольную степенную особенность в нуле. В настоящей работе сформулированы результаты по интегральному представлению решений уравнений с подобными сингулярными потенциалами. От потенциала требуется лишь мажорируемость определенной функцией, суммируемой на бесконечности. В частности, к классу допустимых в данной работе относятся сингулярный потенциал $q=x^{-2}$, сильно сингулярный потенциал со степенной особенностью $q=x^{-2-\varepsilon}, \varepsilon>0$, потенциалы Юкавы типа $q=e^{-\alpha x} / x$, потенциалы Баргмана и Батмана-Шадана [7] и ряд других. При этом на функцию $q(x)$ не накладывается никаких дополнительных условий типа быстрой осцилляции в начале координат или знакопостоянства, что позволяет изучать притягивающие и отталкивающие потенциалы единым методом. Освобождение от ограничивающих условий в нуле - это преимущество рассмотрения операторов преобразования типа Левина (3).

В данной статье основным объектом исследования является интегральное уравнение для ядра оператора преобразования (3). После сведения задачи к интегральному уравнению для него доказаны существование и единственность решения, его необходимая гладкость, получены оценки решения через параметр и потенциал исходного уравнения (1), они выражаются с использованием специальных функций Лежандра. Для частного класса потенциалов степенного вида получены более простые оценки. В работе используется техника, основанная на применении функции Римана для уравнения Эйлера-Пуассона-Дарбу, оценки интегралов с использованием преобразования Меллина и теоремы Слейтер-Маричева.

Следует отметить, что в работе построены операторы преобразования специального вида, отличающиеся от ранее известных некоторыми деталями. До этого рассматривались лишь случаи одинаковых пределов (оба вида $[0 ; a]$ или $[a ; \infty])$ в основном интегральном уравнении для ядра оператора преобразования. В данной работе показано, что можно рассматривать случаи различных пределов в основном интегральном уравнении. Именно такая расстановка пределов и позволила охватить более широкий класс потенциалов с особенностями в нуле. Кроме того, по сравнению с рассуждениями по образцу классической работы Б.М. Левитана [9] мы вносим дополнение и усовершенствование в эту схему, основанные на рассмотрении модифицированного интегрального уравнения для ядра оператора преобразования. Используемую в доказательстве функцию Римана, как оказалось, можно выразить не только через общую гипергеометрическую функцию Гаусса с тремя параметрами, но и более конкретно через функцию Лежандра с двумя параметрами, что позволяет избавиться от неопределенных постоянных в оценках из предыдущих работ. 


\section{2. РЕШЕНИЕ ОСНОВНОГО ИНТЕГРАЛЬНОГО УРАВНЕНИЯ ДЛЯ ЯДРА ОПЕРАТОРА ПРЕОБРАЗОВАНИЯ}

Введем новые переменные и функции по формулам:

$$
\begin{gathered}
\xi=\frac{t+x}{2}, \quad \eta=\frac{t-x}{2}, \quad \xi \geq \eta>0 ; \\
K(x, t)=\left(\frac{x}{t}\right)^{\alpha} P(x, t), \quad u(\xi, \eta)=K(\xi-\eta, \xi+\eta) .
\end{gathered}
$$

Пусть $v=\alpha-1$. Таким образом, для обоснования представления (3) для решения уравнения (1) достаточно определить функцию $u(\xi, \eta)$. Известно [17], [18], что если существует дважды непрерывно дифференцируемое решение $u(\xi, \eta)$ интегрального уравнения

$$
u(\xi, \eta)=-\frac{1}{2} \int_{\xi}^{\infty} R_{v}(s, 0 ; \xi, \eta) q(s) d s-\int_{\xi}^{\infty} d s \int_{0}^{\eta} q(s+\tau) R_{v}(s, \tau ; \xi, \eta) u(s, \tau) d \tau,
$$

при условиях $0<\tau<\eta<\xi<s$, то искомая функция $P(x, t)$ определяется по формулам (5) через это решение $u(\xi, \eta)$. Функция $R_{v}=R_{\alpha-1}$ является функцией Римана, возникающей при решении некоторой задачи Гурса для сингулярного неоднородного гиперболического уравнения вида (одна из форм уравнения Эйлера-Пуассона-Дарбу)

$$
\frac{\partial^{2} u(\xi, \eta)}{\partial \xi \partial \eta}+\frac{4 \alpha(\alpha-1) \xi \eta}{\left(\xi^{2}-\eta^{2}\right)^{2}} u(\xi, \eta)=f(\xi, \eta)
$$

которое в нашем случае преобразуется к виду

$$
\frac{\partial^{2} u(\xi, \eta)}{\partial \xi \partial \eta}+\frac{4 \alpha(\alpha-1) \xi \eta}{\left(\xi^{2}-\eta^{2}\right)^{2}} u(\xi, \eta)=q(\xi+\eta) u(\xi, \eta)
$$

Эта функция Римана известна в явном виде (см. [9], [19]), она выражается через гипергеометрическую функцию Гаусса ${ }_{2} F_{1}$ по формуле

$$
R_{v}=\left(\frac{s^{2}-\eta^{2}}{s^{2}-\tau^{2}} \frac{\xi^{2}-\tau^{2}}{\xi^{2}-\eta^{2}}\right)_{2}^{v} F_{1}\left(-v,-v ; 1 ; \frac{s^{2}-\xi^{2}}{s^{2}-\eta^{2}} \frac{\eta^{2}-\tau^{2}}{\xi^{2}-\tau^{2}}\right)
$$

Это выражение упрощено в [17], где показано, что функция Римана в рассматриваемом случае выражается через функцию Лежандра по формуле

$$
R_{v}(s, \tau, \xi, \eta)=P_{v}\left(\frac{1+A}{1-A}\right), \quad A=\frac{\eta^{2}-\tau^{2}}{\xi^{2}-\tau^{2}} \frac{s^{2}-\xi^{2}}{s^{2}-\eta^{2}} .
$$

Основное содержание статьи составляет

Теорема 1. Пусть функция $q(r) \in C^{1}(0, \infty)$ удовлетворяет условию

$$
|q(s+\tau)| \leq|p(s)|, \quad \forall s, \quad \forall \tau, \quad 0<\tau<s, \quad \int_{\xi}^{\infty}|p(t)| d t<\infty, \quad \forall \xi>0 .
$$

Тогда существует интегральное представление вида (3), ядро которого удовлетворяет оценке

$$
\begin{aligned}
& |P(r, t)| \leq\left(\frac{t}{r}\right)^{\alpha} \frac{1}{2} \int_{\frac{t+r}{2}}^{\infty} P_{\alpha-1}\left(\frac{y^{2}\left(t^{2}+r^{2}\right)-\left(t^{2}-r^{2}\right)}{2 t r y^{2}}\right)|p(y)| d y \times \\
& \times \exp \left[\left(\frac{t-r}{2}\right) \frac{1}{2} \int_{\frac{t+r}{2}}^{\infty} P_{\alpha-1}\left(\frac{y^{2}\left(t^{2}+r^{2}\right)-\left(t^{2}-r^{2}\right)}{2 t r y^{2}}\right)|p(y)| d y\right] .
\end{aligned}
$$

При этом ядро оператора преобразования $P(x, t)$, а также решение уравнения (1) являются дважды непрерывно дифференцируемыми функциями по своим аргументам по отдельности на соответствующих областях определения. 
Доказательство. Разобьем доказательство основной теоремы 1 на ряд лемм.

Введем следующие обозначения:

$$
\begin{gathered}
I_{q}(\xi, \eta)=\frac{1}{2} \int_{\xi}^{\infty} R_{v}(y, 0 ; \xi, \eta)|p(y)| d y=\frac{1}{2} \int_{\xi}^{\infty} P_{v}\left(\frac{y^{2}\left(\xi^{2}+\eta^{2}\right)-2 \xi^{2} \eta^{2}}{y^{2}\left(\xi^{2}-\eta^{2}\right)}\right)|p(y)| d y, \\
u_{0}(\xi, \eta)=-\frac{1}{2} \int_{\xi}^{\infty} R_{v}(s, 0 ; \xi, \eta)|p(s)| d s, \\
\mathbf{A} u_{0}(\xi, \eta)=-\int_{\xi}^{\infty} d s \int_{0}^{\eta} q(s+\tau) R_{v}(s, \tau ; \xi, \eta) u_{0}(s, \tau) d \tau .
\end{gathered}
$$

Докажем равномерную сходимость операторного ряда Неймана

$$
\sum_{k=0}^{\infty} \mathbf{A}^{k} u_{0}(\xi, y)
$$

и возможность его двукратного дифференцирования.

Лемма 1. Справедлива оценка

$$
\left|u_{0}(\xi, \eta)\right| \leq I_{q}(\xi, \eta) .
$$

Доказательство сразу следует из определения (9).

Лемма 2. Пусть $0<\tau<\eta<\xi<s$. Тогда справедливо неравенство

$$
I_{q}(s, t) \leq I_{q}(\xi, \eta)
$$

Доказательство. По условию дано $0<\tau<\eta<\xi<s<y$. Покажем, что тогда

$$
\frac{\tau^{2}}{s^{2}} \frac{\left(y^{2}-s^{2}\right)}{\left(y^{2}-\tau^{2}\right)} \leq \frac{\eta^{2}}{\xi^{2}} \frac{\left(y^{2}-\xi^{2}\right)}{\left(y^{2}-\eta^{2}\right)}
$$

Действительно, это неравенство эквивалентно следующему:

$$
\tau^{2} \xi^{2}\left(y^{2}-s^{2}\right)\left(y^{2}-\eta^{2}\right) \leq \eta^{2} s^{2}\left(y^{2}-\xi^{2}\right)\left(y^{2}-\tau^{2}\right),
$$

которое очевидно, так как каждый из сомножителей слева не превосходит соответствующего сомножителя справа. Далее, рассмотрим при $0<x<1$ функцию

$$
f(x)=\frac{1+x}{1-x} \geq 1, \quad f^{\prime}(x)=\frac{2}{(1-x)^{2}}>0, \quad 0<x<1 .
$$

Следовательно, эта функция возрастает по $x$. Поэтому

$$
\frac{1+\frac{\tau^{2}}{s^{2}} \frac{\left(y^{2}-s^{2}\right)}{\left(y^{2}-\tau^{2}\right)}}{1-\frac{\tau^{2}}{s^{2}} \frac{\left(y^{2}-s^{2}\right)}{\left(y^{2}-\tau^{2}\right)}} \leq \frac{1+\frac{\eta^{2}}{\xi^{2}} \frac{\left(y^{2}-\xi^{2}\right)}{\left(y^{2}-\eta^{2}\right)}}{1-\frac{\eta^{2}}{\xi^{2}} \frac{\left(y^{2}-\xi^{2}\right)}{\left(y^{2}-\eta^{2}\right)}} .
$$

Функция Лежандра $P_{v}(x)$ на интервале $x \in(1, \infty)$ при $v>-1$ монотонно возрастает, кроме того, $P_{v}(x)>1$. Поэтому

$$
P_{v}\left(\frac{1+\frac{\tau^{2}}{s^{2}} \frac{\left(y^{2}-s^{2}\right)}{\left(y^{2}-\tau^{2}\right)}}{1-\frac{\tau^{2}}{s^{2}} \frac{\left(y^{2}-s^{2}\right)}{\left(y^{2}-\tau^{2}\right)}}\right) \leq P_{v}\left(\frac{1+\frac{\eta^{2}}{\xi^{2}} \frac{\left(y^{2}-\xi^{2}\right)}{\left(y^{2}-\eta^{2}\right)}}{1-\frac{\eta^{2}}{\xi^{2}} \frac{\left(y^{2}-\xi^{2}\right)}{\left(y^{2}-\eta^{2}\right)}}\right)
$$

Последнее неравенство можно записать иначе:

$$
P_{v}\left(\frac{y^{2}\left(s^{2}+\tau^{2}\right)-2 s^{2} \tau^{2}}{y^{2}\left(s^{2}-\tau^{2}\right)}\right) \leq P_{v}\left(\frac{y^{2}\left(\xi^{2}+\eta^{2}\right)-2 \xi^{2} \eta^{2}}{y^{2}\left(\xi^{2}-\eta^{2}\right)}\right) .
$$


Заметим, что мы фактически доказали неравенство для функции Римана

$$
R_{v}(y, 0 ; s, \tau) \leq R_{v}(y, 0 ; \xi, \eta)
$$

при условиях $0<\tau<\eta<\xi<s<y$.

Из проведенных вычислений получаем оценку

$$
I_{q}(s, \tau)=\frac{1}{2} \int_{s}^{\infty} R_{v}(y, 0 ; s, \tau)|p(y)| d y \leq \frac{1}{2} \int_{\xi}^{\infty} R_{v}(y, 0 ; s, \tau)|p(y)| d y .
$$

Заменяя нижний предел интегрирования $s$ на $\xi<s$, мы можем лишь увеличить значение интеграла, так как функция Римана положительна, $R_{v}>0$. В результате приходим к оценке (11).

Лемма доказана.

Лемма 3. Для п-го члена ряда Неймана (10) справедлива оценка

$$
\left|u_{n}(\xi, \eta)\right| \leq I_{q}(\xi, \eta) \frac{\left[\eta I_{q}(\xi, \eta)\right]^{n}}{n !} .
$$

Доказательство. Применим метод математической индукции. Для $n=0$ неравенство (13) сводится к уже доказанному неравенству из леммы 1 . Пусть выполнено (13) для некоторого $n=k$. Тогда для очередного члена ряда Неймана получаем

$$
\begin{aligned}
& \left|u_{k+1}(\xi, \eta)\right| \leq\left|\int_{\xi}^{\infty} d s \int_{0}^{\eta} R_{v}(s, \tau ; \xi, \eta) u_{k}(s, \tau) q(s+\tau) d \tau\right| \leq \\
& \leq \int_{\xi}^{\infty} d s \int_{0}^{\eta} R_{v}(s, \tau ; \xi, \eta)|q(s+\tau)| I_{q}(s, \tau) \frac{\left[\eta I_{q}(s, \tau)\right]^{k}}{k !} d \tau .
\end{aligned}
$$

Повторяя рассуждения предыдущей леммы, получаем

$$
R_{v}(s, \tau ; \xi, \eta) \leq R_{v}(s, 0 ; \xi, \eta)
$$

так как

$$
R_{v}(s, \tau ; \xi, \eta)=P_{v}\left(\frac{1+A}{1-A}\right), \quad A=\frac{\eta^{2}-\tau^{2}}{\xi^{2}-\tau^{2}} \frac{s^{2}-\xi^{2}}{s^{2}-\eta^{2}},
$$

а максимальное по $\tau$ значение $A$ достигается при $\tau=0$. Учитывая неравенство (14) и предполагаемое неравенство (13), приходим к оценке

$$
\left|u_{k+1}(\xi, \eta)\right| \leq I_{q}(\xi, \eta) \frac{\left[\tau I_{q}(\xi, \eta)\right]^{k}}{k !} \int_{\xi}^{\infty} R_{v}(s, 0 ; \xi, \eta) \int_{0}^{\eta}|q(s+\tau)| \tau^{k} d \tau d s .
$$

Мы рассматриваем потенциалы, для которых верно неравенство $|q(s+\tau)| \leq|p(s)|, 0<\tau<s$. Окончательно получаем

$$
\left|u_{k+1}(\xi, \eta)\right| \leq I_{q}(\xi, \eta) \frac{\left[I_{q}(\xi, \eta)\right]^{k+1}}{k !} \frac{\eta^{k+1}}{(k+1)}
$$

что и доказывает оценку (13) для всех $n$.

Лемма доказана.

Теперь завершим доказательство теоремы 1. Суммируя все оценки (13), получаем, что ряд Неймана сходится равномерно в области $0<\eta<\xi$, и его сумма есть некоторая непрерывная функция, удовлетворяющая неравенству

$$
|u(\xi, \eta)| \leq I_{q}(\xi, \eta) \exp \left[\eta I_{q}(\xi, \eta)\right] .
$$

Из (15) следует, что мы смогли бы доказать сходимость ряда (10) для суммируемого потенциала $q$, который можно приблизить непрерывными потенциалами. 
Возвращаясь к функциям $K$ и $P$, получаем неравенства

$$
\begin{gathered}
|K(x, t)| \leq I_{q}\left(\frac{t+x}{2}, \frac{t-x}{2}\right) \exp \left[\left(\frac{t-x}{2}\right) I_{q}\left(\frac{t+x}{2}, \frac{t-x}{2}\right)\right], \\
|P(x, t)| \leq\left(\frac{t}{x}\right)^{\alpha} I_{q}\left(\frac{t+x}{2}, \frac{t-x}{2}\right) \exp \left[\left(\frac{t-x}{2}\right) I_{q}\left(\frac{t+x}{2}, \frac{t-x}{2}\right)\right] .
\end{gathered}
$$

Преобразуем величину $I_{q}$, входящую в оценки

$$
I_{q}\left(\frac{t+x}{2}, \frac{t-x}{2}\right)=\frac{1}{2} \int_{\frac{t+x}{2}}^{\infty} P_{\alpha-1}\left(\frac{y^{2}\left(t^{2}+x^{2}\right)-\left(t^{2}-x^{2}\right)}{2 t x y^{2}}\right)|p(y)| d y .
$$

Таким образом, мы приходим к оценке (9).

Для завершения доказательства теоремы 1 осталось обосновать существование вторых непрерывных производных функции $P(x, t)$ по переменным $x, t$ при условии $q \in C^{1}(x>0)$. Очевидно, что это эквивалентно существованию вторых непрерывных производных функции $u(\xi, \eta)$ по переменным $\xi$, $\eta$. Доказательство последнего утверждения проводится по приведенному выше образцу методом последовательных приближений и полностью повторяет соответствующий фрагмент доказательства из [17].

Теорема доказана.

Перечислим классы потенциалов, для которых выполнены условия (8). Если $|q(x)|$ монотонно убывает, то можно принять $p(x)=|q(x)|$. Для потенциалов с произвольной особенностью в начале координат и возрастающих при $0<x<M$ (например, кулоновских $q=-\frac{1}{x}$ ), которые обрезаны нулем на бесконечности, $q(x)=0, x>M$, можно принять $p(x)=|q(M)|, x<M, p(x)=0$, $x \geq M$. Условию (8) будут также удовлетворять потенциалы с оценкой $q(x+\tau) \leq c|q(x)|=|p(x)|$. На возможность подобного усиления теоремы 1 внимание автора обратил В.В. Катрахов.

В частности, приведенным условиям удовлетворяют следующие потенциалы, встречающиеся в приложениях: сильно сингулярный потенциал со степенной особенностью вида $q(x)=x^{-2-\varepsilon}$, различные потенциалы Баргмана

$$
q_{1}(x)=-\frac{e^{-a x}}{\left(1+\beta e^{-a x}\right)^{2}}, \quad q_{2}(x)=\frac{c_{2}}{\left(1+c_{3} x\right)^{2}}, \quad q_{3}(x)=\frac{c_{4}}{c h^{2}\left(c_{5} x\right)},
$$

и Юкавы

$$
q_{4}(x)=-\frac{e^{-a x}}{x}, \quad q_{5}(x)=\int_{x}^{\infty} e^{-a t} d c(t)
$$

(см., например, [7]).

Замечание. Фактически при доказательстве приведенной теоремы не нужен явный вид функции Римана (7). Используются только существование функции Римана, ее положительность и некоторое специальное свойство монотонности (14). Эти факты являются довольно общими, поэтому полученные результаты можно распространить на достаточно широкий класс дифференциальных уравнений.

Оценку из теоремы 1 для потенциалов общего вида можно преобразовать в менее точную, но зато и более обозримую.

Теорема 2. Пусть выполнены условия теоремы 1. Тогда для ядра оператора преобразования $P(x, t)$ справедлива оценка

$$
|P(x, t)| \leq \frac{1}{2}\left(\frac{t}{x}\right)^{\alpha} P_{\alpha-1}\left(\frac{t^{2}+x^{2}}{2 t x}\right) \int_{x}^{\infty}|p(y)| d y \exp \left[\frac{1}{2}\left(\frac{t-x}{2}\right) P_{\alpha-1}\left(\frac{t^{2}+x^{2}}{2 t x}\right) \int_{x}^{\infty}|p(y)| d y\right] .
$$

Отметим, что при $x \rightarrow 0$ ядро интегрального представления может иметь экспоненциальную особенность. 


\section{3. ОЦЕНКИ ДЛЯ СЛУЧАЯ СТЕПЕННОГО СИНГУЛЯРНОГО В НУЛЕ ПОТЕНЦИАЛА}

Для класса потенциалов со степенной сингулярностью вида

$$
q(x)=x^{-(2 \beta+1)}, \quad \beta>0,
$$

полученные оценки можно упростить, не снижая их точности. Ограничение на $\beta$ вызвано условием суммируемости на бесконечности.

Теорема 3. Рассмотрим потенциал вида (16). Тогда теорема 1 выполняется с оценкой

$$
|P(x, t)| \leq\left(\frac{t}{x}\right)^{\alpha} \frac{\Gamma(\beta) 4^{\beta-1}}{\left(t^{2}-x^{2}\right)^{\beta}} P_{\alpha-1}^{-\beta}\left(\frac{t^{2}+x^{2}}{2 t x}\right) \exp \left[\left(\frac{t-x}{x}\right) \frac{\Gamma(\beta) 4^{\beta-1}}{\left(t^{2}-x^{2}\right)^{\beta}} P_{\alpha-1}^{-\beta}\left(\frac{t^{2}+x^{2}}{2 t x}\right)\right],
$$

где $P_{v}^{\mu}(\cdot)-$ функция Лежсандра [20], величина $\beta$ определяется из (16), а величина $\alpha-$ из (2).

Предваряя доказательство, отметим, что данная оценка получается после довольно длинных вычислений с использованием знаменитой теоремы Слейтер-Маричева [21], которая помогает вычислить в терминах гипергеометрических функций необходимые интегралы после их сведения к свертке Меллина.

Доказательство. Для этого класса потенциалов мы упростим оценку (15), составляющую содержание теоремы 1 , не снижая ее точности. Для этого будет вычислена в явном виде величина $I_{q}$, входящая в оценку (15). Доказательство теоремы 3 разобьем на две леммы.

Лемма 4. Для потенциала вида (16) справедливо соотношение

$$
I_{q}(\xi, \eta)=\frac{1}{4 \xi^{2 \beta}} \int_{0}^{1} P_{v}(2 \alpha z+1)(1-z)^{\beta-1} d z
$$

где $P_{v}-$ функция Лежандра, $\alpha=\eta^{2} /\left(\xi^{2}-\eta^{2}\right)$.

Доказательство. Рассмотрим величину

$$
I_{q}(\xi, \eta)=\frac{1}{2} \int_{\xi}^{\infty} P_{v}\left(\frac{t^{2}\left(\xi^{2}+\eta^{2}\right)-2 \xi^{2} \eta^{2}}{t^{2}\left(\xi^{2}-\eta^{2}\right)}\right) \frac{d t}{t^{2 \beta+1}} .
$$

Выполним замену переменных, обозначив аргумент функции Лежандра через $x$,

$$
x=\frac{t^{2}\left(\xi^{2}+\eta^{2}\right)-2 \xi^{2} \eta^{2}}{t^{2}\left(\xi^{2}-\eta^{2}\right)}, \quad d x=\frac{4 \xi^{2} \eta^{2}}{t^{3}\left(\xi^{2}-\eta^{2}\right)} d t .
$$

При такой замене новыми пределами интегрирования станут числа

$$
1, \quad 1+\frac{2 \eta^{2}}{\xi^{2}-\eta^{2}}=\frac{\xi^{2}+\eta^{2}}{\xi^{2}-\eta^{2}}=B>1,
$$

а переменная $t$ будет вычисляться по формуле

$$
t=\xi \eta\left(\frac{2}{\xi^{2}+\eta^{2}-x\left(\xi^{2}-\eta^{2}\right)}\right)^{1 / 2} .
$$

Это приводит к следующему выражению для $I_{q}$ :

$$
I_{q}(\xi, \eta)=\frac{1}{2} \int_{1}^{B} P_{v}(x) \frac{t^{3}\left(\xi^{2}-\eta^{2}\right)}{4 \xi^{2} \eta^{2}} \frac{d t}{t^{2 \beta+1}}=\frac{1}{2} \int_{1}^{B} P_{v}(x)\left[\frac{\xi^{2}-\eta^{2}}{4 \xi^{2} \eta^{2}}\right]\left[\frac{\xi^{2}+\eta^{2}-x\left(\xi^{2}-\eta^{2}\right)}{2 \xi^{2} \eta^{2}}\right]^{\beta-1} d x .
$$

В последнем интеграле выполним еще одну замену переменной по формуле

$$
z=(x-1) \frac{\xi^{2}-\eta^{2}}{2 \eta^{2}}, \quad\left(d z=\frac{\xi^{2}-\eta^{2}}{2 \eta^{2}}\right) d x .
$$


В результате получим

$$
\begin{gathered}
I_{q}(\xi, \eta)=\frac{1}{2}\left(\frac{\xi^{2}-\eta^{2}}{4 \xi^{2} \eta^{2}}\right) \int_{0}^{1} P_{v}(2 \alpha z+1) \frac{2 \eta^{2}}{\xi^{2}-\eta^{2}} \times \\
\times\left[\frac{\xi^{2}+\eta^{2}-\left(\xi^{2}-\eta^{2}\right)\left(\frac{2 \eta^{2}}{\xi^{2}-\eta^{2}} z+1\right)}{2 \xi^{2} \eta^{2}}\right]^{\beta-1} d z=\frac{1}{4 \xi^{2 \beta}} \int_{0}^{1} P_{v}(2 \alpha z+1)(1-z)^{\beta-1} d z,
\end{gathered}
$$

где введено обозначение $\alpha=\eta^{2} /\left(\xi^{2}-\eta^{2}\right)$. Мы получили формулу (17)

Лемма доказана.

Лемма 5. Пусть выполнены условия $а>0, \beta>0$. Тогда справедлива формула:

$$
\int_{0}^{1} P_{v}(2 \alpha x+1)(1-x)^{\beta-1} d x=\Gamma(\beta)\left[\frac{1+\alpha}{\alpha}\right]^{\beta / 2} P_{v}^{-\beta}(2 \alpha+1) .
$$

Доказательство. При доказательстве мы будем использовать обозначения и технику, основанную на теореме Слейтер-Маричева [21].

Произведем в интеграле из (18) замену переменных $t=1 / x$. Получим

$$
\int_{0}^{1} P_{v}(2 \alpha x+1)(1-x)^{\beta-1} d x=\int_{1}^{\infty} P_{v}\left(2 \frac{\alpha}{t}+1\right)(t-1)^{\beta-1} t^{-\beta} \frac{d t}{t}=\int_{0}^{\infty} P_{v}\left(2 \frac{\alpha}{t}+1\right)(t-1)_{+}^{\beta-1} t^{-\beta} \frac{d t}{t}=I(\alpha),
$$

где использовано обозначение для усеченной степенной функции $x_{+}^{\lambda}$. Применим к функции $I(\alpha)$ преобразование Меллина по переменной $\alpha(\alpha>0)$. Используя теорему о свертке Меллина [21], получаем

$$
M[I(\alpha)](s)=M\left[P_{v}(2 x+1)\right](s) M\left[x^{-\beta}(x-1)_{+}^{\beta-1}\right](s) .
$$

Используя последовательно соотношения 6(1), (4), 2(4) из [21], приходим к выражению:

$$
\begin{aligned}
M[I(\alpha)](s) & =-\frac{\sin \pi v}{\pi} \frac{\Gamma(s) \Gamma(-v-s) \Gamma(1+v-s) \Gamma(\beta) \Gamma(1-s)}{\Gamma(1-s) \Gamma(1+\beta-s)}= \\
& =-\frac{\sin \pi v}{\pi} \Gamma(\beta) \Gamma\left[\begin{array}{ll}
s, & -v-s, 1+v-s \\
1+\beta-s
\end{array}\right],
\end{aligned}
$$

где использовано обозначение Слейтер для отношения произведений гамма-функций. В обозначениях теоремы Слейтер-Маричева мы имеем

$$
\begin{gathered}
(a)=(0), \quad(b)=(-v, 1+v), \quad(c)=\varnothing, \quad(d)=(1+\beta), \\
A=1, \quad B=2, \quad C=0, \quad D=1 .
\end{gathered}
$$

Применяя теорему Слейтер-Маричева, получаем формулы для $I(\alpha)$

при $0<\alpha<1$ :

$$
I(\alpha)=-\frac{\sin \pi v}{\pi} \frac{\Gamma(1+v) \Gamma(-v)}{\Gamma(1+\beta)}{ }_{2} F_{1}(-v, 1+v ; 1+\beta ;-\alpha)=\Gamma(\beta) \alpha^{-\beta / 2}(1+\alpha)^{\beta / 2} P_{v}^{-\beta}(1+2 \alpha),
$$

где использована формула (3) из [20, с. 126] и тождество для гамма-функций (см. [20])

$$
\Gamma(-v)=\frac{\pi}{v \Gamma(v) \sin \pi v} .
$$


При $\alpha \geq 1$ получаем по виду другое выражение:

$$
\begin{gathered}
I(\alpha)=-\frac{\sin \pi v}{\pi} \Gamma(\beta)\left\{\alpha^{v} \Gamma\left[\begin{array}{c}
1+v+v, \\
1+\beta+v
\end{array}\right]{ }_{2} F_{1}\left(-v, 1-1-\beta-v ; 1-1-v-v ;-\frac{1}{\alpha}\right)+\right. \\
\left.+\alpha^{-1-v} \Gamma\left[\begin{array}{c}
-v-1-v, 1+v \\
1+\beta-1-v
\end{array}\right]{ }_{2} F_{1}\left(1+v, 1-1-\beta+1+v ; 1+v ;-\frac{1}{\alpha}\right)\right\}= \\
=-\frac{\sin \pi v}{\pi} \Gamma(\beta)\left\{\alpha^{v} \frac{\Gamma(2 v+1) \Gamma(-v)}{\Gamma(1+\beta+v)}{ }_{2} F_{1}\left(-v,-\beta-v ;-2 v ;-\frac{1}{\alpha}\right)+\right. \\
\left.+\alpha^{-1-v} \frac{\Gamma(-1-2 v) \Gamma(1+v)}{\Gamma(\beta-v)}{ }_{2} F_{1}\left(1+v, 1+v-\beta ; 1+v ;-\frac{1}{\alpha}\right)\right\} .
\end{gathered}
$$

Но из [20, с. 131, формула (19)] следует, что выражения, полученные для $I(\alpha)$ при $0<\alpha<1$ и при $\alpha \geq 1$, совпадают.

Из (19) следует, что нами получена искомая формула (18), однако ее вывод не является полностью строгим, так как мы не проверяли законность применения преобразования Меллина и условия справедливости теоремы Слейтер-Маричева (в нашем случае довольно сложные). Однако теперь мы можем применить преобразование Меллина к обеим частям полученного формального равенства (18). В результате доказываем, что при $\alpha>0, \beta>0$ соотношение (18) является тождеством.

Лемма доказана.

Как следствие, теперь получаем нужную оценку для теоремы 3.

Теорема доказана.

Простейшая подобная оценка была получена в работе [17] для потенциала $q(x)=c x^{-2}$, для которого $\beta=\frac{1}{2}$. Как следует из [20], в этом случае функция Лежандра $P_{v}^{-1 / 2}(z)$ может быть выражена через элементарные функции. Поэтому и соответствующая оценка может быть выражена через элементарные функции.

Другим потенциалом, для которого полученная оценка может быть еще упрощена и выражена через элементарные функции, является потенциал вида $q(x)=x^{-(2 \beta+1)}$, когда параметры связаны соотношением $\beta=\alpha-1$.

Следствие. Пусть выполнено соотношение между параметрами $\beta=\alpha-1$. Тогда оценка из теоремы 3 принимает вид

$$
\begin{gathered}
|P(x, t)| \leq\left(\frac{t}{x}\right)^{\beta+1} \frac{2^{\beta-2}}{\beta}\left[\frac{t^{2}+x^{2}}{2 t x}\right]^{\beta} \exp \left[\left(\frac{t-x}{2}\right) \frac{2^{\beta-2}}{\beta}\left[\frac{t^{2}+x^{2}}{2 t r}\right]^{\beta}\right]= \\
=\frac{1}{4 \beta} \frac{1}{x^{2 \beta+1}}\left(t^{2}+x^{2}\right)^{\beta} \exp \left[\frac{2^{\beta-2}}{\beta}\left(\frac{t-x}{2}\right)\left(\frac{t^{2}+x^{2}}{2 t x}\right)^{\beta}\right] .
\end{gathered}
$$

Доказательство. В этом случае преобразуем оценку из теоремы 3 к виду

$$
\begin{gathered}
\frac{\Gamma(\beta) 4^{\beta-1}}{\left(t^{2}-x^{2}\right)^{\beta}} P_{\beta}^{-\beta}\left(\frac{t^{2}+x^{2}}{2 t x}\right)=\frac{\Gamma(\beta) 4^{\beta-1}}{\left(t^{2}-x^{2}\right)^{\beta}} \frac{2^{-\beta}}{\Gamma(\beta+1)}\left[\left(\frac{t^{2}+x^{2}}{2 t x}\right)^{2}-1\right]^{\beta / 2}= \\
=\frac{2^{\beta-2}}{\beta} \frac{1}{\left(t^{2}-x^{2}\right)^{\beta}} \frac{\left(t^{2}-x^{2}\right)^{\beta}\left(t^{2}+x^{2}\right)^{\beta}}{(2 t x)^{\beta}}=\frac{2^{\beta-2}}{\beta}\left[\frac{t^{2}+x^{2}}{2 t x}\right]^{\beta},
\end{gathered}
$$

где при преобразованиях использована формула (см. [20])

$$
P_{v}^{-v}(z)=\frac{2^{-v}}{\Gamma(v+1)}\left(z^{2}-1\right)^{v / 2}, \quad z>1 .
$$

Поэтому неравенство для ядра при $\beta=\alpha-1$ принимает вид (20).

Следствие доказано. 
Отметим, что при $\alpha=0$ в формулах (1), (2), теорема 1 сводится к известным оценкам для ядра интегрального представления типа Левина для уравнения Штурма-Лиувилля.

Изложенная техника полностью переносится и на задачу о построении неклассических операторов обобщенного сдвига. Данная задача по существу эквивалентна выражению решений уравнения

$$
B_{\alpha, x} u(x, y)-q(x) u(x, y)=B_{\beta, y} u(x, y)
$$

через решения невозмущенного уравнения Эйлера-Пуассона-Дарбу с операторами Бесселя по каждой переменной (в несингулярном случае - волнового) при наличии дополнительных условий, обеспечивающих корректность. Такие представления получаются уже из факта существования операторов преобразования и изучались для несингулярного случая $(\alpha=\beta=0)$ в [22], [23] как следствия теории обобщенного сдвига. Интересная оригинальная методика для получения подобных представлений также в несингулярном случае разработана в работах А.В. Боровских [24]. Из результатов настоящей работы следуют интегральные представления некоторого подкласса решений уравнения (22) в общем сингулярном случае для достаточно произвольных потенциалов с особенностями в начале координат. При этом оценки для решений не содержат никаких неопределенных постоянных, а для ядер интегральных представлений в явном виде выписываются интегральные уравнения, которым они удовлетворяют.

\section{4. АСИМПТОТИЧЕСКИ ТОЧНЫЕ НЕРАВЕНСТВА ДЛЯ ФУНКЦИЙ ЛЕЖАНДРА}

В заключение покажем, как можно использовать формулу (18), полученную при выводе леммы 5, для установления асимптотически точной нижней оценки для функций Лежандра. Эта оценка, на наш взгляд, представляет самостоятельный интерес.

Рассмотрим очевидные неравенства $(\alpha>0)$ :

$$
\int_{0}^{1} P_{v}(1+2 \alpha x)(1-x)^{-1 / 2} d x \leq \int_{0}^{1} P_{v}(1+2 \alpha x) x^{-1 / 2}(1-x)^{-1 / 2} d x \leq P_{v}(1+2 \alpha) \int_{0}^{1} x^{-1 / 2}(1-x)^{-1 / 2} d x .
$$

Первый интеграл получается из формулы (18) при $\beta=\frac{1}{2}$, он был вычислен в лемме 5. Второй интеграл является известным, и, как следует, например, из [25, с. 225], вычисляется по формуле

$$
I=\pi^{1 / 2} \Gamma\left(\frac{1}{2}\right)\left\{P_{v}(\sqrt{1+\alpha})\right\}^{2} .
$$

Следовательно, справедливо неравенство

$$
\sqrt{\pi} \alpha^{-1 / 4}(\alpha+1)^{-1 / 4} P_{v}^{-1 / 2}(2 \alpha+1) \leq \pi\left\{P_{v}(\sqrt{1+\alpha})\right\}^{2} .
$$

Выражая функцию Лежандра через элементарные функции, получаем окончательно

$$
\left\{P_{v}(\sqrt{1+\alpha})\right\}^{2} \geq \frac{1}{\pi(2 v+1) \sqrt{x}}\left\{(\sqrt{x}+\sqrt{x+1})^{2 v+1}-(\sqrt{x}+\sqrt{x+1})^{-2 v-1}\right\} .
$$

Доказанное неравенство дает правильную асимптотику по всем входящим в него параметрам, как это следует из асимптотической формулы, приведенной в [26, с. 107]

$$
\left[P_{v}(\sqrt{1+x})\right]^{2} \sim \frac{1}{2 \pi v} \frac{(\sqrt{x}+\sqrt{x+1})^{2 v+1}}{\sqrt{x}} .
$$

Второе из рассмотренных интегральных неравенств приводит также к интересной оценке

$$
\left\{P_{v}(\sqrt{1+\alpha})\right\}^{2} \leq P_{v}(1+2 \alpha)
$$

В связи с последними приведенными неравенствами заметим, что функции Лежандра связаны с полными и неполными эллиптическими интегралами Лежандра трех родов, эти два класса специальных функций при определенных значениях выражаются друг через друга. С другой стороны, функции Лежандра - это частные случаи гипергеометрической функции Гаусса. Различные неравенства для эллиптических интегралов Лежандра рассматриваются в совместных работах автора [27], [28], а неравенства для гипергеометрических функций - в работах [29], [30].

В заключение автор выражает благодарность А.В. Глушаку (Белгородский государственный национальный исследовательский университет “НИУ БелГУ”, Белгород, Россия) и В.В. Кра- 
вченко (CINVESTAV, Керетаро, Мексика) за полезные замечания, которые позволили уточнить постановку рассматриваемой задачи.

\section{СПИСОК ЛИТЕРАТУРЫ}

1. Carroll R. Transmutation, Scattering Theory and Special Functions. Elsevier, North Holland, 1982.

2. Carroll R. Transmutation Theory and Applications. Elsevier, North Holland, 1986.

3. Марченко B.A. Операторы Штурма-Лиувилля и их приложения. Киев: Наук. Думка, 1977.

4. Левитан Б.М. Обратные задачи Штурма-Лиувилля. М.: Наука, 1984.

5. Катрахов B.B., Ситник C.M. Метод операторов преобразования и краевые задачи для сингулярных эллиптических уравнений // Современная математика. Фундаментальные направления. 2018. Т. 64. № 2. C. $211-426$.

6. Ситник С.М., Шишкина Э.Л. Метод операторов преобразования для дифференциальных уравнений с операторами Бесселя. М.: Физматлит, 2019.

7. Шадан К., Сабатье П. Обратные задачи в квантовой теории рассеяния. М.: Мир, 1980.

8. Hryniv R.O., Mykytyuk Ya.V. Transformation Operators for Sturm-Liouville Operators with Singular Potentials. Dedicated to Professor V.A. Marchenko on the occasion of his 80th birthday // Math. Physics, Analysis and Geometry. 2004. V. 7. P. 119-149.

9. Левитан Б.М. Разложения по функциям Бесселя в ряды и интегралы Фурье // Успехи матем. наук. 1951. Т. 6. Вып. 2. С. 102-143.

10. Сташевская В.В. Об обратной задаче спектрального анализа для дифференциального оператора с особенностью в нуле // Уч. зап. Харьковского матем. об-ва. 1957. № 5. С. 49-86.

11. Сохин А.С. Об одном классе операторов преобразования // Тр. физ.-тех. ин-та низких температур АН УССР. 1969. Вып. 1. С. 117-125.

12. Сохин А.С. О преобразовании операторов для уравнений с особенностью специального вида // Вестник Харьковского университета. 1974. № 113. С. 36-42.

13. Kravchenko V.V. A representation for solutions of the Sturm-Liouville equation // Complex Variables and Elliptic Equations. 2008. V. 53. P. 775-789.

14. Kravchenko V.V., Porter R.M. Spectral parameter power series for Sturm-Liouville problems // Math. Method App. Sci. 2010. V. 33. P. 459-468.

15. Kravchenko V.V. On a method for solving the inverse scattering problem on the line // Math. Methods in the Applied Sciences. 2019. V. 42. P. 1321-1327.

16. Kravchenko V.V. On a method for solving the inverse Sturm-Liouville problem // J. Inverse and Ill-Posed Problems. 2019. V. 27. P. 401-407.

17. Ситник С.М. Оператор преобразования и представление Йоста для уравнения с сингулярным потенциалом // Препринт ИАПУ ДВО РАН. Владивосток, 1993. 21 с.

18. Катрахов В.В., Ситник С.М. Оценки решений Йоста одномерного уравнения Шрёдингера с сингулярным потенциалом // Докл. АН СССР. 1995. Т. 340. № 1. С. 18-20.

19. Килбас A.A., Маричев О.И., Репин О.A. Краевые задачи для уравнений в частных производных с разрывными коэффициентами. Самара: Изд-во Самарского государственного экономического университета, 2008.

20. Бэйтмен Г., Эрдейи А. Высшие трансцендентные функции. М.: Наука, 1973. Т. І.

21. Маричев О.И. Метод вычисления интегралов от специальных функций. Минск: Наука и техн., 1978.

22. Левитан Б.М. Применение операторов обобщенного сдвига к линейным дифференциальным уравнениям второго порядка // Успехи матем. наук. 1949. № 4:1 (29). С. 3-112.

23. Левитан Б.М. Операторы обобщенного сдвига и некоторые их применения. М.: Физматлит, 1962.

24. Боровских А.В. Метод распространяющихся волн // Труды семинара имени И.Г. Петровского. 2004. Вып. 24. С. 3-43.

25. Бэйтмен Г., Эрдейи А. Таблицы интегральных преобразований. М.: Наука, 1970. Т. І.

26. Полиа Г., Сеге Г. Задачи и теоремы из анализа. М.: ГИТТЛ, 1956.

27. Karp D.B., Sitnik S.M. Asymptotic approximations for the first incomplete elliptic integral near logarithmic singularity // J. Computational and Applied Mathematics. 2007. V. 205. P. 186-206.

28. Karp D., Savenkova A., Sitnik S.M. Series expansions for the third incomplete elliptic integral via partial fraction decompositions // J. Computational and Applied Mathematics. 2007. Vol. 207. Issue 2. P. 331-337.

29. Karp D., Sitnik S.M. Inequalities and monotonicity of ratios for generalized hypergeometric function // J. Approximation Theory. 2009. V. 161. P. 337-352.

30. Karp D.B., Sitnik S.M. Log-convexity and log-concavity of hypergeometric-like functions // J. Mathematical and Applications. 2010. V. 364. P. 384-394. 